$$
f_{i}^{(n)}(t), f_{i}^{(n+1)}(t), \cdots, f_{i}^{(n+q-1)}(t)
$$

plus a sum of $q$-rowed determinants each of which has at least one column consisting of the derivatives of $f_{1}(t), f_{2}(t)$, $\cdots, f_{q}(t)$ of an order less than $n$.

Using this fact we find upon differentiating the determinant $\Delta\left(\lambda_{1}+1\right)\left(\lambda_{2}+1\right)$ times with respect to $t_{2}$ and putting $t_{2}=t_{1}$ that the result is equal to a positive integer multiplied by the $p$-rowed determinant whose $i$ th row is

$$
\begin{aligned}
f_{i}\left(t_{1}\right), f_{i}{ }^{\prime}\left(t_{1}\right), \cdots, f_{i}^{\left(\lambda_{1}\right)}\left(t_{1}\right), f_{i}{ }^{\left(\lambda_{1}+1\right)}\left(t_{1}\right), \cdots, f_{i}{ }^{\left(\lambda_{1}+\lambda_{2}+1\right)}\left(t_{1}\right), \\
f_{i}\left(t_{3}\right), \cdots, f_{i}^{\left(\lambda_{3}\right)}\left(t_{3}\right), \cdots, \cdots, f_{i}\left(t_{\mu}\right), \cdots, f_{i}{ }^{\left(\lambda_{\mu}\right)}\left(t_{\mu}\right) .
\end{aligned}
$$

The other determinants which result from the differentiation drop out when $t_{2}$ is put equal to $t_{1}$, since each of them then has at least two columns identical. Repeating this process for the other variables in turn, we finally have the $p$-rowed determinant whose $i$ th row is

$$
f_{i}\left(t_{1}\right), f_{i}{ }^{\prime}\left(t_{1}\right), \cdots, f_{i}{ }^{(p-1)}\left(t_{1}\right)
$$

(or the wronskian of the $p$ given functions) vanishing identically if the determinant of the theorem does. The necessity of the condition of the theorem follows immediately as in the proof concerning the wronskian.

HARVARD UNIVERSITY,

July, 1916.

\title{
ON THE LINEAR DEPENDENCE OF FUNCTIONS OF ONE VARIABLE.
}

\author{
BY DR. G. M. GREEN.
}

(Read before the American Mathematical Society, September 5, 1916.)

As is well known, the identical vanishing of the wronskian of $p$ functions of a single variable is a sufficient condition for their linear dependence if the functions are analytic; if, however, they are not analytic the vanishing of the wronskian is not sufficient. The same situation arises in connection with the theorem proved by Mr. Morse and by Dr. Pfeiffer in the present number of the BuLletin. The former makes explicit use of the analytic character of the functions involved, whereas the theorem proved by the latter may be neatly stated only for analytic functions, if it is to afford a criterion for linear dependence. 
The purpose of the present note is to establish a sufficient condition under which the vanishing of the determinant of Morse and Pfeiffer implies linear dependence, in the case of non-analytic functions - real or complex - of a real variable. The proof is like Frobenius's modification for the wronskian theorem, as given by Bôcher.* The theorem for analytic functions of a real or complex variable follows immediately from the general one.

The following seems to be the most convenient form in which to state the theorem for non-analytic functions, although the hypothesis is stronger than it need be. The superscripts of course denote differentiation with respect to the arguments indicated.

Let $f_{1}(t), f_{2}(t), \cdots, f_{p}(t)$ be $p$ functions of the real variable $t$ defined on the interval

$$
\text { I: } \quad a \leqq t \leqq b \text {, }
$$

and possessing in I all derivatives appearing in the determinant

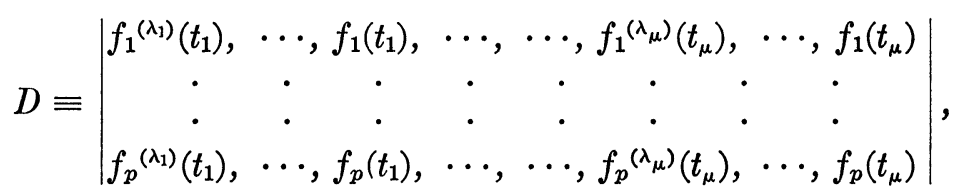

where $t_{1}, t_{2}, \cdots, t_{\mu}$ are independent variables on the interval $I$. Suppose that

$1^{\circ}$. There exist a set of values of $t_{2}, t_{3}, \cdots, t_{\mu}$ for which the $(p-1)$-rowed determinant formed by deleting the first column and the last row of $D$ vanishes for no value of $t_{1}$ in $I$, and

$2^{\circ}$. For the said values of $t_{2}, t_{3}, \cdots, t_{\mu}$ the determinant $D$ vanishes for every value of $t_{1}$ in $I$.

Then the functions $f_{1}(t), f_{2}(t), \cdots, f_{p}(t)$ are linearly dependent in $I$, and in fact

$$
f_{p}(t)=c_{1} f_{1}(t)+c_{2} f_{2}(t)+\cdots+c_{p-1} f_{p-1}(t) .
$$

We shall suppose throughout the discussion that the set of values of $t_{2}, t_{3}, \cdots, t_{\mu}$ mentioned in the statement of the theorem

* "Certain cases in which the vanishing of the wronskian is a sufficient condition for linear dependence," Transactions Amer. Math. Society, vol. 2 (1901), pp. 139-149, Theorem II.

Professor Osgood noticed that Bôcher's proof could be extended to the theorem in question; without knowing of this, the present writer sent the proof given below to Professor Osgood, who insisted upon its publication. 
have been fixed once for all. It will now be convenient to use a new notation, writing $D$ in the form

$$
D \equiv\left|\begin{array}{cccc}
u_{1}^{(1)}, & u_{1}^{(2)}, & \cdots, & u_{1}^{(p)} \\
u_{2}^{(1)}, & u_{2}^{(2)}, & \cdots, & u_{2}^{(p)} \\
\cdot & \cdot & \cdot & \cdot \\
\cdot & \cdot & \cdot & \cdot \\
u_{p}^{(1)}, & u_{p}^{(2)}, & \cdots, & u_{p}^{(p)}
\end{array}\right|,
$$

each element standing for the element in the corresponding place in the original determinant. Here the superscripts of course do not denote differentiation.

Let $D_{i}$ denote the cofactor of $u_{i}^{(1)}$ in $D$. Then by part $1^{\circ}$ of the hypothesis $D_{p}$ is zero for no value of $t_{1}$ in $I$. It is immediately evident that

(1) $u_{1}{ }^{(i)} D_{1}+u_{2}{ }^{(i)} D_{2}+\cdots+u_{p}{ }^{(i)} D_{p}=0 \quad(i=1,2, \cdots, p)$,

for every $t_{1}$ in $I$. From these relations may be obtained as follows the $p-1$ relations, for every $t_{1}$ in $I$ :

$$
u_{1}{ }^{(i)} D_{1}{ }^{\prime}+u_{2}{ }^{(i)} D_{2}{ }^{\prime}+\cdots+u_{p}{ }^{(i)} D_{p}{ }^{\prime}=0 \quad(i=2,3, \cdots, p),
$$

where the accents on the $D$ 's denote differentiation with respect to $t_{1}$. Those of equations (2) for which $i>\lambda_{1}+1$ follow at once from differentiation of the corresponding equations (1), since, if $i>\lambda_{1}+1, u_{1}{ }^{(i)}, u_{2}{ }^{(i)}, \cdots, u_{p}{ }^{(i)}$ are all constants. To obtain the first $\lambda_{1}$ equations of the set (2), differentiate with respect to $t_{1}$ each of the first $\lambda_{1}+1$ of equations (1), except the first, and subtract from the result the equation immediately preceding it.

Now add equations (2), after multiplying the first of them by the cofactor of $u_{1}^{(2)}$ in $D_{p}$, the second by the cofactor of $u_{1}^{(3)}$ in $D_{p}$, etc., the last by the cofactor of $u_{1}{ }^{(p)}$ in $D_{p}$. The result is the following equation, true for every value of $t_{1}$ in $I$ :

$$
D_{1}{ }^{\prime} D_{p}-D_{p}{ }^{\prime} D_{1}=0 \text {. }
$$

Since $D_{p}$ is by hypothesis zero for no value of $t_{1}$ in $I$, we may divide through equation (3) by $D_{p}{ }^{2}$. This gives the equation

$$
\frac{\partial}{\partial t_{1}}\left(\frac{D_{1}}{D_{p}}\right)=0 \text {, }
$$

for every value of $t_{1}$. Recalling that the values of $t_{2}, t_{3}$, 
$\cdots, t_{\mu}$ have been fixed, we may therefore infer that

$$
D_{1}=-c_{1} D_{p} \text {, }
$$

where $c_{1}$ is a constant. In the same way we find that

$$
D_{2}=-c_{2} D_{p}, \cdots, \cdots, D_{p-1}=-c_{p-1} D_{p-1} .
$$

Since the $\left(\lambda_{1}+1\right)$ th column of $D$ consists of the elements $f_{1}\left(t_{1}\right), \cdots, f_{p}\left(t_{1}\right)$, we have the identity in $t_{1}$

$$
D_{1} f_{1}\left(t_{1}\right)+D_{2} f_{2}\left(t_{1}\right)+\cdots+D_{p} f_{p}\left(t_{1}\right)=0,
$$

which by the relations obtained above may be written

$$
D_{p}\left(-c_{1} f_{1}\left(t_{1}\right)-c_{2} f_{2}\left(t_{1}\right)-\cdots-c_{p-1} f_{p-1}\left(t_{1}\right)+f_{p}\left(t_{1}\right)\right)=0 \text {. }
$$

Then, since for the fixed values of $t_{2}, t_{3}, \cdots, t_{\mu}$ the factor $D_{p}$ is different from zero for every value of $t_{1}$ in $I$, the expression in parentheses must vanish identically in $t_{1}$. This proves the theorem.

An obvious weakening of the hypothesis may be made if any of the $\lambda$ 's be greater than $\lambda_{1}$. In the statement of the theorem, we supposed that all of the derivatives appearing in $D$ exist throughout $I$. Of course all that need be required, if derivatives of order higher than $\lambda_{1}$ appear in $D$, is that such derivatives exist at discrete points in $I$, constituted by some or all of the fixed values $t_{2}, t_{3}, \cdots, t_{\mu}$ mentioned in part $1^{\circ}$ of the hypothesis.

The statement and proof of the general theorem, though given above only for a real independent variable, hold also without any essential modification when the functions are analytic and the independent variable complex. From the theorem for this case may be proved at once the theorem given by Mr. Morse, viz., that if $D$ vanishes identically when considered as a function of the $\mu$ independent variables $t_{1}, t_{2}$, $\cdots, t_{\mu}$, the analytic functions $f_{1}(t), f_{2}(t), \cdots, f_{p}(t)$ are linearly dependent. For, if $D_{p}$ is not identically zero, there must be a set of values of the independent variables, say $t_{1}, \bar{t}_{2}, \cdots, \bar{t}_{\mu}$, for which $D_{p}$ is different from zero. Then for the fixed values $\bar{t}_{2}, \bar{t}_{3}, \cdots, \bar{t}_{\mu}, D_{p}$ is nowhere zero in a certain neighborhood about the point $\bar{t}_{1}$, but $D$ is identically zero in this neighborhood. Therefore by the general theorem the functions are linearly dependent in this neighborhood, and, being analytic, are linearly dependent throughout their common region of definition. If, however, $D_{p}$ is identically zero in all its argu- 
ments, we may proceed afresh, since $D_{p}$ is of the same form as $D$, but contains only the functions $f_{2}, f_{3}, \cdots, f_{p}$. Reasoning as above, we find that these $p-1$ functions are linearly dependent, and hence also the $p$ given functions, unless the $(p-2)$-rowed determinant in the upper right-hand corner of $D_{p}$ vanishes identically. In the latter case we must continue in the same way, until we finally reach a determinant in the upper right-hand corner of $D$ which is not identically zero. But there must be one such, unless $f_{p}(t)$ is itself identically zero, in which case the given $p$ functions are linearly dependent. The theorem for analytic functions as stated in Mr. Morse's note therefore follows.

It may be of interest to point out that many-if not allof the theorems on linear dependence in which wronskians or determinants and matrices constructed like wronskians are involved* have their analogues in corresponding theorems in which appear determinants and matrices resembling the determinant $D$.

HARVARD UNIVERSITY, July, 1916.

\title{
TRANSLATION SURFACES ASSOCIATED WITH LINE CONGRUENCES.
}

\author{
BY PROFESSOR O. E. GIENN.
}

(Read before the American Mathematical Society, October 28, 1916.)

\section{§1. Introduction.}

IN a note published in the BuLLETIN in 1914 $\uparrow$ I established an algorism on a class of surfaces associated with line congruences in 3-space, which result by translation from invariants of plane $n$-lines. It is the purpose of this paper to apply symbolical methods to the study of some properties of these surfaces.

Two non-homogeneous forms of respective orders $m, n$, in Plücker's line coordinates $p_{1}, p_{2}, q_{1}, q_{2}, r[r=(p q)]$, considered together, represent a congruence $(m, n)$. For the sake of symmetry let the variables be changed to the homogeneous system

\footnotetext{
* Such, for instance, as are given by Bôcher, loc. cit., and by Curtiss, Math. Annalen, vol. 65 (1908), pp. 282-298.

$\dagger$ Bulletin, vol. 20 , p. 233.
} 